\title{
Characteristics of a Two-Body Holonomic Constraint Mechanical System
}

\author{
Haiduke Sarafian'1, Nenette Hickey ${ }^{2}$ \\ ${ }^{1}$ The Pennsylvania State University: University College, York, PA, USA \\ ${ }^{2}$ Pennsylvania Department of Health: Division of TB/STD, Harrisburg, PA, USA \\ Email: has2@psu.edu, Nenette.hickey@gmail.com
}

How to cite this paper: Sarafian, H. and Hickey, N. (2017) Characteristics of a TwoBody Holonomic Constraint Mechanical System. World Journal of Mechanics, 7, 161166.

https://doi.org/10.4236/wjm.2017.76014

Received: May 9, 2017

Accepted: May 24, 2017

Published: May 27, 2017

Copyright ( 92017 by authors and Scientific Research Publishing Inc. This work is licensed under the Creative Commons Attribution International License (CC BY 4.0).

http://creativecommons.org/licenses/by/4.0/

\begin{abstract}
Two massive blocks are connected with a massless unstretchable line of $2 \ell$. One of the masses is placed on a horizontal frictionless table, $\ell$ distance away from the edge of the table the other one is held horizontally equidistance from the edge along the extension of the line. The latter is released from rest. As it falls under gravity's pull, it drags the one on the table. It is the interest of this investigation to analyze the kinematics of the system. Because of the holonomic constraint of the system, analysis of the problem encounters complicated super nonlinear coupled differential equations. Utilizing Mathematica we solve the equations numerically. Applying the solutions we quantify numerous kinematic quantities; most interestingly we evaluate the run-time, and the trajectory of the falling block. Analysis is robust allowing us to address the "what if" scenarios.
\end{abstract}

\section{Keywords}

Computer Algebra System, Mathematica, Holonomic Constraint, Mechanical System

\section{Motivation and Goals}

The investigation of the proposed scenario outlined in the abstract stems from questioning, "For two identical blocks which one reaches the vertical leg of the table first?" [1] [2]. Intuitively, it is an easy task without quantifying... Claim the block on the table! Quantification of the run-time, however, requires analytic analysis. It requires developing either expressions for the horizontal displacements of the blocks or their numeric plots vs. time. A holonomic [3] two-coordinate dependent constraint of the problem via an explicit time-independent relationship between the coordinates i.e. the length of the line, complicates the analysis. It is trivial to set up the equations of motion; solving them simulta- 
neously encounters a set of extraordinary non-linear coupled differential equations. Applying a Computer Algebra System (CAS), specifically Mathematica [4], [5] provides numeric solutions. Having the solutions in hand quantitatively we confirm our intuitive claim. In addition to determining numerous kinematic quantities, we identify the trajectory of the falling body. We address the proposed quantities by dividing the article in three sections. Section 2 embodies the layout of the physics of the problem including a derivation of the needed equations of motion, their solutions and the results that are mostly graphics. Section 3 is the conclusion and a few suggestions to augment the scope of the analysis addressing the "what if scenarios".

\section{Physics of the Problem}

Figure 1 shows the setup of the problem. Two identical blocks are connected with a massless, unstretchable line of length $2 \ell$. Block 2 rests on the frictionless horizontal table top and the second one, block 1, is held horizontally along the extension of the line, equidistance from the edge. Conveniently the origin of the coordinate system is set as shown, with the vertical axis downward. At any given instance the coordinates of the blocks are, $\left(x_{2}(t), 0\right)$ and $\left(x_{1}(t), y_{1}(t)\right)$, respectively.

Block 1 is released from rest, as it falls pulls on block 2 . The falling block sets a time-dependent tension, $T(t)$, in the line. The massless line holds the same tension in the line. Tension, $T$, and angle, $\theta$, with vertical are shown.

Applying Newton's law gives the equations of motion for the blocks,

$$
\begin{gathered}
T \sin (\theta)=m \ddot{x}_{1}, \\
m g-T \cos (\theta)=m \ddot{y}_{1},
\end{gathered}
$$

And

$$
T=m \ddot{x}_{2},
$$

The holonomic constraint is formulated as,

$$
2 \ell=x_{2}(t)+\sqrt{x_{1}(t)^{2}+y_{1}(t)^{2}},
$$

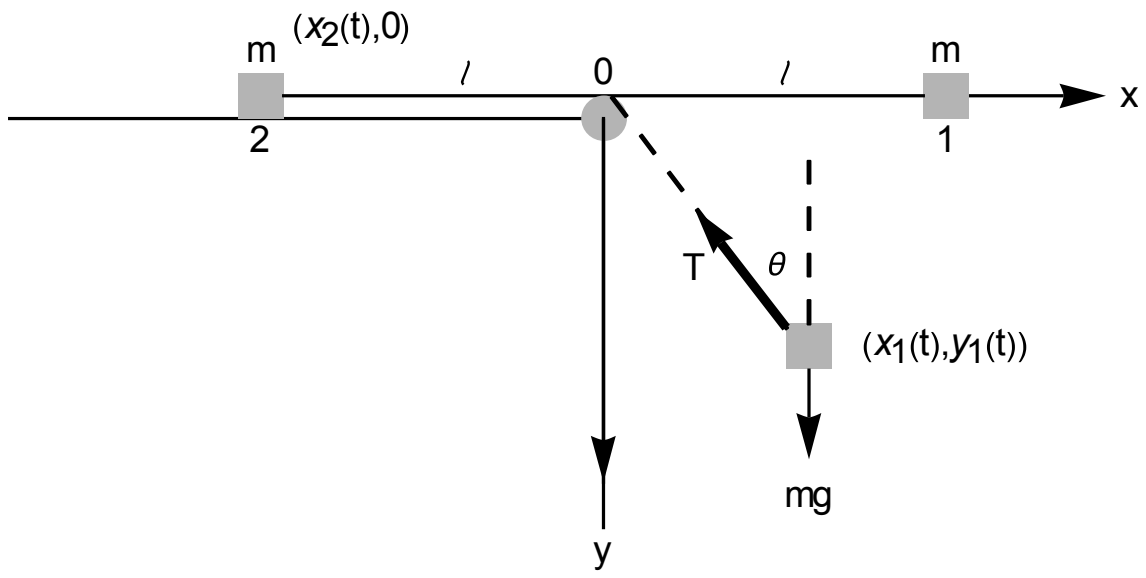

Figure 1. Setup of the problem. 
From Equations (1) and (3) and substituting for $\sin \theta=\frac{x_{1}}{\sqrt{x_{1}^{2}+y_{1}^{2}}}$, we deduce,

$$
\frac{1}{\sqrt{x_{1}(t)^{2}+y_{1}(t)^{2}}}=\frac{1}{x_{1}(t)} \frac{\ddot{x}_{1}(t)}{\ddot{x}_{2}(t)}
$$

On the other hand from Equations (2)-(4) and substituting for

$$
\cos \theta=\frac{y_{1}}{\sqrt{x_{1}^{2}+y_{1}^{2}}}
$$

we have,

$$
g-\frac{y_{1}(t)}{x_{1}(t)} \ddot{x}_{1}(t)=y_{1}(t)
$$

Equation (6) depends only to the coordinates of block 1.

By the same token Equation (5) is reduced to the coordinates of the block 1 provided $x_{2}(t)$ is replaced applying constraint Equation (4),

$$
\begin{aligned}
& \frac{x_{1}(t)}{\sqrt{x_{1}(t)^{2}+y_{1}(t)^{2}}} \\
= & \frac{\ddot{x}_{1}(t)}{\left[x_{1}(t) \dot{x}_{1}(t)+y_{1}(t) \dot{y}_{1}(t)\right]^{2}}-\frac{\dot{x}_{1}(t)^{2}+\dot{y}_{1}(t)^{2}+x_{1}(t) \ddot{x}_{1}(t)+y_{1}(t) \ddot{y}_{1}(t)}{\left[x_{1}(t)^{2}+y_{1}(t)^{2}\right]^{\frac{3}{2}}}
\end{aligned}
$$

Now Equation (7) depends only on the coordinates of block 1.

Equations (6) and (7) form a set of coupled ordinary differential equations. Hopelessly we were unable to solve the set of Equations (6) and (7) analytically. We apply Mathematica's numeric solver NDSolve; we were stunned with its power! Coordinates of the blocks are shown in Figure 2.

For practical reasons the half-length of the line is set $l=1.0 \mathrm{~m}$. Because the blocks are identical, derived equations are mass independent. To set the time

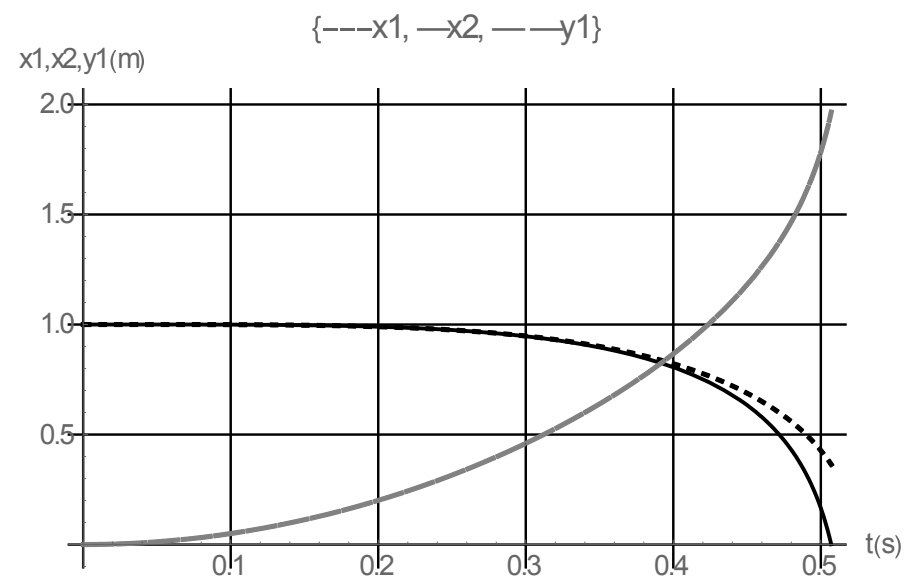

Figure 2. Coordinates of block 1 and 2 vs. $t$. The dashed, and gray lines are $x_{1}(t), y_{1}(t)$; the solid black line is the $x_{2}(t)$. 
scale we apply the free-fall run-time, namely $t=\sqrt{2 \frac{l}{g}}=0.45 \mathrm{~s}$.

The run-time of the actual case not being a free fall is a bit longer. Figure 2 is the display of the coordinates of the blocks vs. time. Accordingly, as intuitively expected, block 2 , the one that slides on the table (solid curve) reaches the edge first; this figure confirms our intuition. At the beginning blocks are one meter away from the edge; their distances change and coordinates as shown change accordingly. Block 1 lags behind, so that when block 2 is at the edge, block 1 is not quite there. The length of the line is $2.0 \mathrm{~m}$; the end tip of the solid gray curve is not quite $2.0 \mathrm{~m}$ showing the connecting line is not perfectly vertical.

By eliminating parameter $t$ between the coordinates of block 1, it enables us to display its trajectory shown in Figure 3.

As shown in Figure 3 by the end of the run-time block 1 horizontally is off from the edge of the vertical leg and has fallen $1.8 \mathrm{~m}$. Another interesting geometrical quantity is the time-dependent profile of the $\theta(t)$. The plot of this function, $\theta=\arcsin \left[\frac{x_{1}}{\sqrt{x_{1}^{2}+y_{1}^{2}}}\right]$, vs. $t$ is shown in Figure 4 .

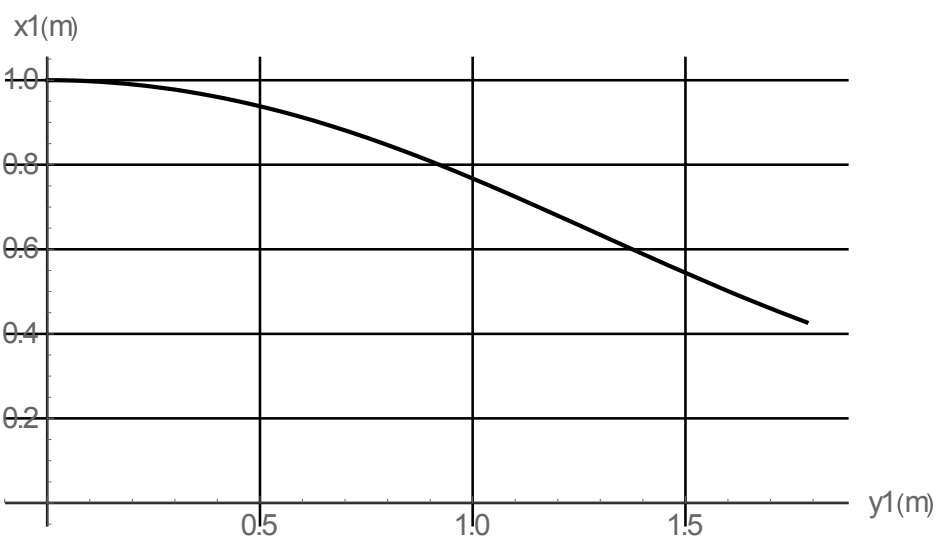

Figure 3. Trajectory of the block 1 .

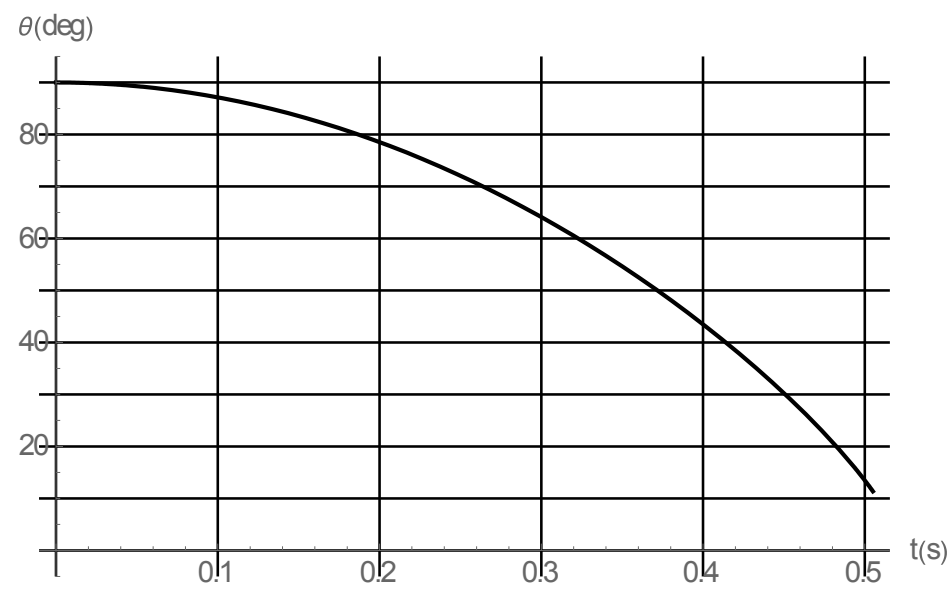

Figure 4. Display of orientation of the hanging line with respect to vertical position, i.e. $\theta$, vs. $t$. 
As shown, $\theta$, begins at $90^{\circ}$ and at the end of run-time plunges to about $11^{\circ}$.

Next we utilize the solutions evaluating kinematic quantities such as speed and acceleration of the individual block. These are displayed in Figure 5.

Figure 5(a) shows beyond a certain instance, say about 0.4 s, block 2 (solid) moves faster than block 1 (dashed). The same is true for their associated accelerations depicted in Figure 5(b). These are additional justifications quantifying why block 2 should reach the edge first.

Figure $6(\mathrm{a})$ is the vertical acceleration of block 1. Figure $6(\mathrm{~b})$ is the tension vs. time.

As depicted the falling block begins with gravity acceleration, g, gains acceleration as it falls, Figure 6(a). Had we considered a simple pendulum scenario with a constant length pivoted at the edge, the acceleration of the block at the bottom would have been $2 \mathrm{~g}$.

Applying Equation (1) we also depict the tension in the line, $T$; this is shown in Figure 6(b). For the sake of simplicity we assumed $m=1.0 \mathrm{~kg}$. As shown the tension at the beginning is zero. While the block falls tension builds reaching to its maximum value, over valuing the static limit of $10.0 \mathrm{~N}$ by a factor nine.

We complete the analysis by displaying one of many phase diagrams, namely the plot of horizontal speed of block 1 vs. its horizontal coordinate, i.e. $\left(x_{1}(t), \dot{x}_{1}(t)\right)$.

Figure 7 shows the falling block while at its farthest distance from the edge begins with a zero horizontal speed reaching its maximum speed at closest distance to the edge.

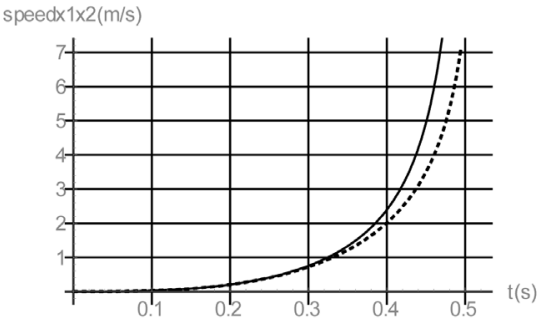

(a)

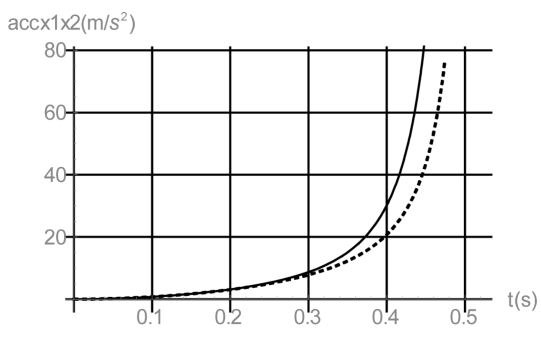

(b)

Figure 5. (a) is the plot of the horizontal speeds of the blocks; block 1 is dashed, block 2 is solid; (b) is the display of their associated accelerations.

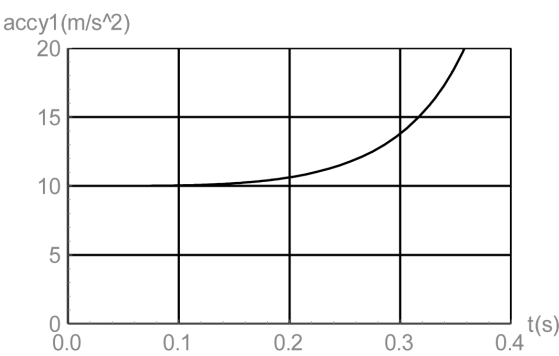

(a)

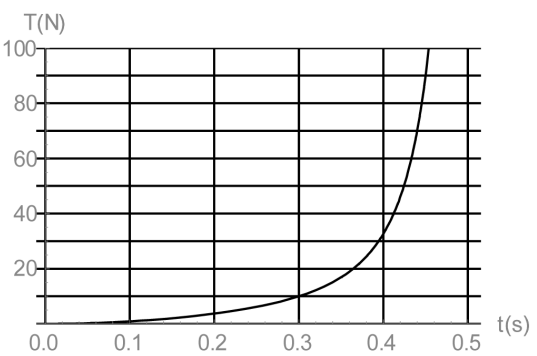

(b)

Figure 6. Vertical acceleration of block 1 (a) and tension (b) vs. time. 


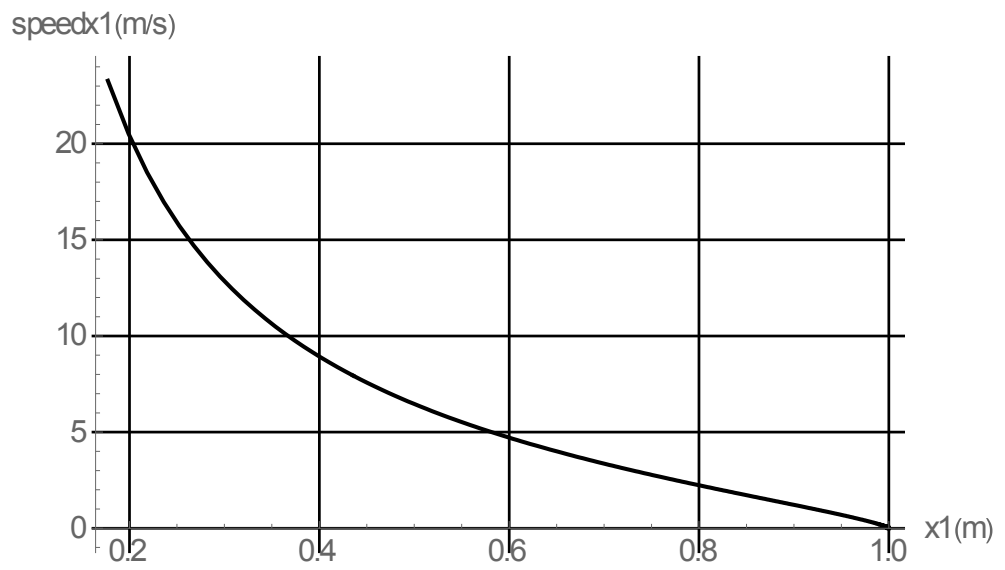

Figure 7. Phase diagram of horizontal speed of block 1 vs. its horizontal coordinate.

\section{Conclusions and Suggestions}

We analyze a problem that on its face is trivial. Although true, its detailed analysis is proven to be challenging. The challenge stems from the fact that the solution of the needed equations being a set of coupled super nonlinear ordinary differential equations analytically is unsolvable. This is another example that one needs to apply a Computer Algebra System (CAS). By applying one of the most powerful CASs, Mathematica, amazingly we were able to deduce the needed solutions. Having the solutions on hand, various information, e.g. trajectory of the falling object, dynamic quantities e.g. tension in the line is evaluated. For the majority of the quantities of interest we also applied the superb graphic capabilities of Mathematica displaying the results. An interested reader may extend the scope of the investigation considering 1) non-identical masses 2) determining masses making the blocks reach the edge simultaneously and 3) replacing the massless line with a massive one. Reference [6] embodies Mathematica codes and information needed to produce the graphs embedded in this article.

\section{References}

[1] Halliday, D., Resnick, R. and Walker, J. (2014) Fundamental of Physics. 10th Edition, Wiley and Sons, New York.

[2] Prokopenya, A.N. (2017) Motion of a Swinging Atwood's Machine: Simulation and Analysis with Mathematica. Mathematics in Computer Science, 1-9. https://doi.org/10.1007/s11786-017-0301-9

[3] Gantmacher, F. (1970) Lectures in Analytical Mechanics. MIR Publications, Moscow.

[4] Mathematica ${ }^{\mathrm{Tm}}$ (2015) Is Symbolic Computation Software. V11.0, Wolfram Research Inc.

[5] Wolfram, S. (1996) Mathematica Book. 3rd Edition, Cambridge University Press, Cambridge.

[6] Sarafian, H. (2015) Mathematica Graphics Example Book for Beginners. Scientific Research Publishing. http://www.scirp.org 
Submit or recommend next manuscript to SCIRP and we will provide best service for you:

Accepting pre-submission inquiries through Email, Facebook, LinkedIn, Twitter, etc. A wide selection of journals (inclusive of 9 subjects, more than 200 journals)

Providing 24-hour high-quality service

User-friendly online submission system

Fair and swift peer-review system

Efficient typesetting and proofreading procedure

Display of the result of downloads and visits, as well as the number of cited articles Maximum dissemination of your research work

Submit your manuscript at: http://papersubmission.scirp.org/

Or contact wjm@scirp.org 